\title{
Aprendizaje multimedia y transferencia de conocimiento en una plataforma digital. Estudio de caso de Entremedios
}

\section{(Multimedia Learning and Knowledge Transfer on a Digital Platform. Entremedios Case Study)}

\author{
Carmen Marta-Lazo \\ José Antonio Gabelas-Barroso \\ Antonia Nogales-Bocio \\ Universidad de Zaragoza (España) \\ Miguel Ezequiel Badillo-Mendoza \\ Universidad Nacional Abierta y a Distancia (Colombia)
}

DOI: https://doi.org/10.5944/ried.25.1.30846

\section{Cómo referenciar este artículo:}

Marta-Lazo, C., Gabelas-Barroso, J. A., Nogales-Bocio, A., Badillo-Mendoza, M. E. (2022). Aprendizaje Multimedia y Transferencia de Conocimiento en una Plataforma Digital. Estudio de Caso de Entremedios. RIED. Revista Iberoamericana de Educación a Distancia, 25(1), pp. 101-120. https://doi. org/10.5944/ried.25.1.30846

\section{Resumen}

En la sociedad digital, la sociabilidad conectada se produce a través de "plataformas", que conjuntan las dimensiones técnica y simbólica. Estos medios sociales permiten la oportunidad de comunicarse, interactuar o intercambiar productos y servicios. Las llamadas TRIC (Tecnologías de la Relación, Información y Comunicación), ponen en valor el Factor Relacional que se produce en cualquier proceso educomunicativo, en el que las interacciones permiten desarrollar el análisis, la reflexión y el pensamiento crítico, entre los co-autores, a través de la comunicación horizontal y la construcción colaborativa. En la última década, se han llevado a cabo varias actividades que han tenido como objetivo implementar, en varios cursos de grado y posgrado, el aprendizaje atendiendo a una perspectiva holística desde una dimensión multimedia. Como metodología de análisis, planteamos el estudio de caso de la plataforma digital Entremedios, a través de la triangulación de grupos de discusión a egresados y entrevistas en profundidad a responsables. A través de los resultados obtenidos, pudimos constatar los aspectos mejorables, tales como la ampliación de los contenidos, la mayor periodicidad y la mayor profesionalización, con fines de transferencia de conocimiento de quienes se preparan para ser periodistas en la Universidad de Zaragoza. Como conclusión, 
se plantea terminar de vertebrar la interacción con los usuarios para aplicar en mayor medida la filosofía relacional del medio.

Palabras clave: TRIC; transferencia; plataforma digital; aprendizaje multimedia; entremedios.

\begin{abstract}
In the digital society, connected sociability occurs through "platforms", which combine the technical and symbolic dimensions. These social media allow the opportunity to communicate, interact or exchange products and services. The so-called ICRT (Relationship, Information and Communication Technologies), value the Relational Factor that occurs in any educommunicative process, in which the interactions allow the development of analysis, reflection and critical thinking, among the co-authors, through horizontal communication and collaborative construction. In the last decade, several activities have been carried out that have aimed to implement, in various undergraduate and graduate courses, learning from a holistic perspective using a multimedia dimension. As an analysis methodology, we propose the case study of the digital platform Entremedios, through the triangulation of discussion groups with graduates and in-depth interviews with managers. We were able to verify the aspects that could be improved through the results obtained, such as the expansion of the contents, greater frequency and greater professionalization, for the purpose of knowledge transfer of those who are preparing to become journalists at the University of Zaragoza. In conclusion, it is proposed to finish structuring the interaction with users in order to apply the relational philosophy of the medium to a greater extent.
\end{abstract}

Keywords: ICRT; transfer; digital platform; multimedia learning; entremedios.

El debate sobre la pérdida de la condición humana en un contexto de conectividad automatizada está vivo y necesitado de análisis, no exento de riesgos y dificultades. Las plataformas tecnológicas fueron a comienzos de este siglo, un espacio horizontal, abierto y descentralizado, pero en las dos últimas décadas ha sufrido un notable giro en su centralización y control, no solo de contenidos, también del capital social que se produce, lo cual cuestiona hacia dónde vamos y quiénes controlan el rumbo. Por consiguiente, es obligado el análisis de la naturaleza y calidad de las interacciones que se activan en el universo digital. Es necesario potenciar una línea de investigación que cuestione el ámbito TIC, y que incluya la irrupción de las plataformas tecnológicas (en particular desde inicios del siglo $\mathrm{XX}$ ) en la comunicación, información, entretenimiento y educación, mediados por la tecnología.

Desde esta perspectiva, Van Dijk define los medios conectivos como parte sustancial de un "ecosistema tecno-cultural de carácter cambiante, donde pueden apreciarse tendencias y también tensiones e inconsistencias, que permiten proyectar distintos escenarios futuros" (Van Dijk, 2016, p. 11). El paso de la comunicación en red a la sociabilidad de las plataformas significa una codificación de las conexiones 
humanas. Van Dijk analiza ese complejo proceso de codificación, que convierte las actividades humanas en actos formales, gestionables y manipulables. De este modo, es muy posible conducir las relaciones cotidianas y sociales de los usuarios, que acaecen en la mediación tecnológica.

La inteligencia artificial ha constituido un escenario 4.0 en el que el machine learning es un factor dominante en la Red, que no por casualidad disfruta del beneplácito del tejido empresarial y del Big Tech. Establece cuál es la única opción para mejorar cualquier comportamiento humano en el que medie la tecnología, sea de un sector u otro. No es fácil encontrar una crítica. Sin embargo, uno de los sesgos más utilizados por Google es el de selección, como se puede observar en determinadas rutas de datos que, tal y como demuestra Murray (2020), desbordan el valor casuístico. Pongamos por ejemplo una búsqueda elemental como "European art”, en la que previamente cualquiera puede pensar en Velázquez, Picasso, Monet, Goya, etc. Si ustedes hacen la prueba, la primera imagen catalogada por Google es "People of color european art". Con un básico conocimiento de la historia del arte, sabemos que los retratos de personas negras conforman una muy reducida parte de la historia del arte europeo.

La máquina ha sido entrenada para que esta haya sido la búsqueda. No es necesario llegar a otro sitio, la historia se convierte en un atrezzo de un presente establecido, la plataforma en su bien marcada línea de circulación señala lo que existe y lo que existió. Habrá que dedicar un rato más a nuestra búsqueda para encontrar bastantes otras muchas y representativas muestras del arte europeo, que retratan o muestran el trabajo de artistas que no son negros.

El estar en las redes sociales significa ser crítico no solo en la lectura y escritura en la Red, también ser competentes en la identificación de sesgos inconscientes, que pueden incrementar la desconfianza en nuestros instintos, que incluso pueden impulsar un aprendizaje que reprograme nuestros comportamientos, actitudes y puntos de vista preexistentes. O sea, nuestros sentimientos, nuestras ideas, esa particular cosmovisión que cada ciudadano tiene de la vida es cuestionable, pero no por cada individuo, sino por el llamado aprendizaje automático justo (machine learning fairness), que filtra y a su vez impide que los seres humanos podamos emitir juicios, dado que siempre serán imperfectos, mejorables, si no son conformes a los patrones previamente diseñados.

Para comprender el fenómeno de la sociabilidad conectada hay que precisar el término "plataforma", que reúne dos dimensiones: la técnica y la simbólica. Las plataformas son medios sociales "no necesariamente porque permiten que se escriba o corra un código, sino porque brindan una oportunidad de comunicarse, interactuar o vender" (Gillespie, 2010, p. 351). Por consiguiente, es un término que comprende aspectos computacionales y arquitectónicos, pero en un sentido simbólico y figurativo también contiene una dimensión sociocultural. 


\section{Aprendizaje multimedia y nuevos entornos formativos}

Las fórmulas y los entornos para el aprendizaje online se han venido desarrollando a lo largo de las últimas décadas persiguiendo, sobre todo, la implementación de recursos y la optimización de la experiencia del usuario. Esto, además de influir en una notable cantidad de investigaciones en torno al aprendizaje online (Mayer, 2018), también ha alumbrado estudios acerca de la influencia del entorno virtual en el proceso formativo y en las propias actitudes del estudiantado en comparación con el aprendizaje presencial (Hurlbut, 2018; Nennig et al., 2020). Estos nuevos entornos propician también la aparición de nuevos roles y perfiles que se tornan fundamentales en el online learning. La figura del moderador se convierte no solo en una herramienta para el control de calidad sino también para la orientación y tutorización a lo largo de todo el proceso de aprendizaje. Una labor de formación técnica complementaria que puede repercutir incluso en que las calificaciones finales obtenidas por el alumnado sean mejores (Hurlbut, 2018). Ese es el papel que adoptan, como veremos, los jefes de redacción en "Entremedios" y los redactores jefe y coordinadores en Radio Unizar. También, en cuanto al filtrado de calidad, los profesores gestores de la plataforma "Entremedios".

En cuanto al aprendizaje multimedia, Bidarra y Rusman (2017, p. 13), destacan que "los estudiantes que crean portafolios e historias digitales mejoran sus habilidades utilizando programas informáticos que combinan una variedad de herramientas multimedia que permiten operar con texto, imágenes fijas, audio, vídeo y publicación en la web". Las formas multimedia pueden así contribuir a optimizar el proceso de aprendizaje, dotando al estudiantado de un papel proactivo y asignándole un rol de productor de contenidos e información, algo que empodera al discente y lo implica en el proceso de formación de manera real. Esto resulta especialmente pertinente en los contextos de aprendizaje en los que están presentes los jóvenes representantes de la "Generación Net" (Bullen y Morgan, 2015), estudiantes tan inmersos en un mundo interconectado digitalmente que tienen formas y expectativas diferentes sobre el proceso de aprender.

Asimismo, el aprendizaje multimedia mediante la creación de productos puede servir para construir el conocimiento de forma activa y crítica a través de la adquisición de las competencias mediáticas fundamentales: "los procesos sociales horizontales, la colaboración y la interacción potencian su contacto [el del estudiantado] con los medios de comunicación hasta el punto de ser capaces de crear sus propios productos multimedia" (Marta-Lazo et al., 2016, p. 15). Un paso más allá del multimedia, en tanto que integración de recursos digitales para la producción de contenidos, está el transmedia: expansión de una historia utilizando diferentes medios (SaavedraBautista et al., 2017). La narrativa transmedia es una estrategia de comunicación aplicable al mundo educativo universitario, dado que "es una técnica de elaboración de mensajes, una estrategia de comunicación multiplataforma y multiformato que involucra necesariamente una diversidad de elementos para confeccionar 
los mensajes que se quieren transmitir" (Ossorio-Vega, 2014, p. 3). Los mensajes originales a transmitir pueden ser conocimientos de distintas ramas del saber pero enriquecidos con el carácter transmedia, esto es, potenciando "la participación del alumnado y la necesidad de reforzar su autosuficiencia y su capacidad crítica, al exigirles seleccionar el camino a seguir, discriminar información, ampliar lo ofrecido y, en definitiva, convertirse en protagonistas de su propio proceso de aprendizaje" (Ossorio-Vega, 2014, p. 15).

En este contexto cobran especial relevancia los entornos virtuales de aprendizaje, los cuales cumplen un rol innovador en el proceso de enseñanza y posibilitan el surgimiento de estrategias que avecinan un nuevo paradigma. Los entornos virtuales de aprendizaje son "espacios formativos que para innovar en la docencia requieren utilizar metodologías centradas en el alumno, cambio de roles en docentes y estudiantes, incorporar las TIC para colaborar, compartir y distribuir conocimiento en red" (Silva, 2017, p. 2). Especialmente a nivel universitario, destaca su papel como apoyo a la docencia presencial, b-learning o e-learning, que no debe limitarse a un traspaso al espacio virtual del modelo formativo convencional.

En este tipo de espacios, "los estudiantes se encuentran interconectados y pueden apropiarse de nuevos conocimientos generados en procesos de análisis y reflexión colaborativa y superar la posición de simples espectadores del mensaje" (Cerdeño-Romero, 2019). Para ello, también es preciso que el docente adquiera una serie de competencias que le permitan desarrollar funciones tanto técnicas, como pedagógicas, de orientación, de diseño de materiales didácticos adaptados, moderación de actividades online, tutorización, seguimiento, dinamización y moderación de foros virtuales, etc. No solo resulta prioritario el manejo de nuevas herramientas, sino también, como plantea la filosofía del Factor Relacional, la producción de aprendizaje y la gestación de experiencias compartidas. Estos escenarios digitales de aprendizaje se concretan de distintas formas y se pueden complementar, lo que ofrece diferentes utilidades (Salinas, 2004). Pueden ir desde la gestión online de cualquier actividad formativa, hasta el aprovechamiento e implementación de redes sociales y espacios cuya innovación es la explotación de tecnologías avanzadas, como la realidad virtual (Gisbert y Johnson, 2015). Todo ello permite crear realidades y experiencias centradas en el usuario y dotar al estudiante de una mayor capacidad de actuación.

\section{METODOLOGÍA}

La elección del estudio de caso dentro de la investigación educativa ha sido reforzada desde el punto de vista teórico y metodológico en la literatura científica. Numerosos autores destacan la versatilidad del método (Angulo y Vázquez, 2003; Coller, 2005) y la aplicabilidad de sus resultados a la hora de plantear nuevos escenarios de acción, gestión y formación. Para algunos autores, "los casos de investigación son especialmente valiosos porque permiten el estudio de la causalidad 
y la plasman en una teoría (...), su potencialidad radica en su capacidad para generar premisas hipotéticas y orientar la toma de decisiones" (Álvarez y San Fabián, 2012, p. 2).

En este sentido, el estudio de caso es, para Cebreiro López y Fernández Morante (2004), especialmente procedente cuando se abordan problemas complejos o dinámicas en las que la relación con el contexto juega un papel esencial, tanto que la disociación resultaría contraproducente a los objetivos de investigación: "cuando el objeto que se quiere indagar está difuso, es complejo, escurridizo o controvertido. Es decir, para analizar aquellos problemas o situaciones que presentan múltiples variables y que están estrechamente vinculados al contexto en el que se desarrollan" (Cebreiro López y Fernández Morante, 2004, p. 667).

Álvarez y San Fabián (2012) destacan las tres fases que comprende el desarrollo del estudio de caso como método principal: una fase preactiva, en la que se recopilan los fundamentos epistemológicos, los objetivos, criterios de selección, recursos, técnicas, cronograma de actuación, etc. en torno al problema o caso que se estudia; la fase interactiva, centrada en la recogida, reducción y relación de información a través de diferentes técnicas, tales como observación participante, entrevista, foros de debate y análisis documental; y la fase posactiva, concerniente a la elaboración del informe final en el que se detallan las reflexiones críticas sobre el problema o el caso analizado. A la plasmación de esta última fase corresponde el presente artículo.

Por último, conviene destacar que en los estudios de caso es posible también poner en práctica fórmulas que permitan determinar el impacto de una innovación en el proceso de aprendizaje. La satisfacción y las actitudes de los estudiantes pueden medirse a través de protocolos de recogida de datos, incluidas las calificaciones de los estudiantes, o los instrumentos de percepción de la instrucción por parte de estos (Dziuban et al., 2018). En el presente artículo, se recurrirá a las opiniones de estudiantes para contrastar la información y las percepciones acerca de la plataforma "Entremedios". El guion utilizado tanto en las entrevistas en profundidad, como en el grupo de discusión, fueron validados por expertos en metodología de investigación cualitativa.

El grupo de discusión estuvo conformado por una muestra de nueve ex alumnos/ as del Grado en Periodismo de la Universidad de Zaragoza, cuatro chicos y cinco chicas, con edades comprendidas entre los 24 y los 30 años y pertenecientes a tres promociones distintas de estos estudios.

El guion de contenidos que abordamos en el grupo de discusión atendió a los siguientes parámetros:

- La plataforma Entremedios como medio de aprendizaje: debilidades y fortalezas.

- Relación de las secciones con los contenidos aprendidos en las diferentes asignaturas de la titulación.

- La plataforma Entremedios como medio de comunicación: oportunidades y amenazas. 
- Canales de proyección externa de la plataforma: nuevas posibilidades.

- Planteamiento de posibles mejoras de Entremedios a corto plazo.

Las entrevistas en profundidad se realizaron a los dos profesores doctores que se encuentran al frente de la plataforma "Entremedios", el co-director y la responsable de la sección de Comunicación Digital.

Los aspectos que abordamos en la entrevista en profundidad atendieron a los siguientes apartados:

- Filosofía de trabajo de Entremedios.

- La herramienta como medio de aprendizaje práctico y proyección pública de los futuros periodistas.

- Enfoque de la plataforma como medio de comunicación: contenidos y audiencia.

- Futuras líneas de implementación.

\section{El Factor Relacional en la órbita de sus referentes}

La necesidad de cuestionar y ofrecer otro enfoque, planteamiento y metodología de trabajo que han abordado las tradicionales TIC, abrió un debate que surgió en el primer trimestre del año 2012, en el marco de la primera edición del Máster de Redes Sociales y Aprendizaje Digital de la UNED, en concreto en el seno de la asignatura "Principios de Comunicación Digital". En marzo de 2012, apareció el término TRIC (Tecnologías de la Relación, Información y Comunicación), publicado por primera vez con el título "Por qué las TRIC y no las TIC", en el que se integra la R como "Factor Relacional" que dota de consistencia y sentido a las TRIC. A partir de entonces, con más detalle, cuerpo y continuidad se desarrolló en diferentes plataformas: Habitaciones de Cristal, Sinapsile, INED21. A inicios de 2013, se lanzó la primera plataforma procedente de las TRIC denominada TRICLab y el 18 enero de 2017 se lanzó el sitio con una gran repercusión en las redes sociales, siendo TT en España, la tarde-noche de ese día, que posteriormente se convirtió en https://triclab. es/. Estos últimos nueve años han sido muy fructíferos en el desarrollo de esta línea, que en el "I Simposio Internacional sobre Brecha Digital" realizado por el grupo de investigación Conincom-Austica, en la Facultad de Comunicación Blanquerna de la Universidad Ramon LLull, se proyecta la importancia del Factor Relacional con la siguiente conclusión: "El factor Relacional del uso tecnológico adolescente y joven. Para los jóvenes las redes sociales son un espacio de socialización vital y necesario donde aprenden habilidades, destrezas y saberes básicos necesarios para desarrollarse como ser humano competente: conocerse, presentarse, trabajar la identidad, compartir gustos o aficiones".

La experimentación con el Factor R como clave de la renovación de las TIC continúa su desarrollo en 2013 con la segunda edición del Máster de Redes Sociales y Aprendizaje Digital de la UNED. En coherencia con la filosofía abierta del concepto 
TRIC, las prácticas de los alumnos de dos de las asignaturas, "Principios de la Comunicación Digital" y "Educación 2.0", toman este parámetro como principio y método de trabajo. Los resultados de la línea de investigación TRIC volverán a ser objeto de debate en Barcelona los días 14 y 15 de noviembre de 2013, en la segunda edición del Congreso Internacional de Educación Mediática y Competencia Digital, organizado por la Universitat Oberta de Catalunya. También tendrá un protagonismo en los congresos del CICID 2014, 2015, 2017 y 2019, así como en el III Congreso Internacional de Educación Mediática que se celebró en julio de 2017 en Segovia (España). A lo largo de estos últimos años, muchas han sido las publicaciones de impacto que han recogido estos términos en su creciente línea de investigación.

El Factor Relacional recoge una dimensión estructural de nodos, conexiones y relaciones, que es un valor interdisciplinar que contiene y afecta a materias tan diversas como la educación, neurociencia, comunicación, filosofía, psicología social, y que vertebra tres ámbitos tradicionalmente separados como son la educación, la salud y la comunicación. Por consiguiente, nos encontramos ante un enfoque que además de ecléctico, es sumativo y abierto, puesto que está en construcción. Se observa cómo la analogía entre la propia red global (Internet), la red interna (cerebro), y la red social ("Sociedad-Red") se retroalimentan en una permanente e intensa conexión.

"TRIC es un acrónimo de Tecnologías dela Relación, Información y Comunicación que se presenta como una revisión, superación y desmitificación del reduccionismo tecnológico del término TIC" (Marta-Lazo y Gabelas, 2016, p. 27), en referencia a las Tecnologías de la Información y la Comunicación. Surge en defensa de un entorno virtual que promueve la comunicación horizontal y la alfabetización mediática para configurar modelos educativos basados en la construcción colaborativa de la inteligencia colectiva y en la concepción del internauta no solo como un receptor, sino también como un emisor, creador y coautor.

La línea de investigación que contiene el Factor Relacional (Gabelas-Barroso et al., 2013) recoge fundamentos teóricos de la "cultura de la participación" (Jenkins, 2007) y la "Sociedad Red" (Castells, 2006), además de la larga trayectoria de los estudios en Media Ecology.

Las TIC han objetivado su análisis en la tecnología como instrumento, subestimando el potencial de la comunicación y conformando la información como conjunto de datos. La órbita de referentes citados ha cimentado una línea de investigación denominada Factor Relacional, entendido como:

el eje que conecta los usos, consumos e interacciones que se producen en las redes, tanto psicosociales como sinápticas, con una visión positiva y holística, que abarca las tres dimensiones del ser humano (cognitiva, emocional y social), que desde el desarrollo comunitario contempla todos los agentes mediadores sociales en un triple entorno (individual, social y ambiental) (Gabelas-Barroso y Marta-Lazo, 2017, p. 21). 
Es urgente desplegar el mapa no solo que ubique a cada uno frente a la información que recibimos, también que oriente en el actual laberinto de constante y agresiva estimulación, que obtura con más frecuencia de lo que parece, la capacidad de análisis y crítica.

El potencial relacional asigna un valor subsidiario a la tecnología, trabaja la conversión del dato de la información en conocimiento, y potencia la comunicación. Contempla la tecnología como entorno, en el que el sujeto relacional filtra, contrasta y procesa la información, desde una comunicación que interacciona con los contenidos y los usuarios que participan.

\section{Aplicación del Factor Relacional a la plataforma digital "Entremedios"}

En el curso académico 2015-2016, se creó la plataforma multimedia "Entremedios", diseñada para que fuera un espacio en el que los estudiantes del Grado en Periodismo de la Universidad de Zaragoza, pudieran alojar sus prácticas y mejores trabajos, al mismo tiempo que les permitiera visibilizar sus competencias periodísticas. En su diseño, entendimos que era importante que pudieran ejercer el periodismo de manera real mientras se estaban formando, pudiendo mostrar a través de la Red sus mejores prácticas, con el fin de poder interactuar y conseguir retroalimentación con los usuarios y seguidores.

Dado que el Factor Relacional ya disponía de una relevante trayectoria en su desarrollo teórico y una sustancial praxis en diferentes escenarios presenciales, semipresenciales y online (Marta-Lazo et al., 2016), se planteó que fuera la cimentación conceptual para este embrionario proyecto. De este modo, en los siguientes tres años, su dimensional relacional se proyecta en la construcción de las diferentes secciones que componen el medio, así como en la dinámica de trabajo entre sus colaboradores, públicos y contenidos.

En el curso 2019-2020, se revisan y replantean algunos aspectos sustanciales de la plataforma "Entremedios". Se detecta la necesidad de diseñar un salto cualitativo en los contenidos, donde "Entremedios" es un medio de comunicación de los estudiantes para el público, que es la sociedad. El carácter abierto de los contenidos es una extraordinaria motivación para que los estudiantes de periodismo ejerzan como periodistas y cuenten a la sociedad los problemas y las realidades que les preocupan desde las diferentes secciones que dispone la plataforma. Esto obliga a que los contenidos respondan a las expectativas de la sociedad, y que los estudiantes elaboren piezas en las que "la realidad se toca", con reporterismo a pie de calle, cuestionando la información en los diferentes sectores.

Para conseguir esta calidad es necesario categorizar el producto, para lo cual se establecen unos criterios de selección en la gestación y elaboración de contenidos de cada sección, se crean unas figuras en los equipos de redacción de cada sección (el jefe de sección), que revisará los contenidos, para pasar el segundo control de calidad desde la editora, que finalmente será validado por el profesor que coordina cada 
sección. Se redacta un sencillo libro de estilo, ahora mismo en proceso de revisión y reedición.

Se aumenta la frecuencia de publicación en el número de artículos en cada sección. Hasta el curso 2018-2019 la periodicidad era semanal, que se ha convertido en diaria. Con un detallado calendario de publicaciones, cada sección asumió la publicación de una pieza semanal. Como la plataforma está estructurada en siete secciones, todos los días de la semana se publicó una pieza. Programación que se mantuvo desde septiembre 2019 hasta el mes de abril 2020, que quedó suspendida temporalmente debido a la pandemia.

En el curso 2020-2021, se retomó este plan de producción, diseñando unas líneas y mecánicas de trabajo online en las diferentes redacciones de cada sección. En septiembre, se llevó a cabo un primer seminario con los profesores coordinadores de sección para retomar estas líneas y concretar la mecánica de trabajo.

El Factor Relacional ha sido el motor de este proyecto en el marco del escenario pos-TIC, que denominamos TRIC, "Tecnologías de la Relación, la Información y la Comunicación". La doble dimensión del Factor Relacional cristaliza en este proyecto. Cada redacción está formada por entre cuatro y seis estudiantes, varios son los colaboradores, uno de ellos es el jefe de sección, otro ejerce de editor, más un profesor que es el coordinador de sección. A su vez, hemos creado una coordinación técnica cuya responsable es una becaria de cuarto curso del Grado en Periodismo.

Las secciones estarán conducidas por los dos directores de la plataforma, la profesora Carmen Marta-Lazo, y el profesor José Antonio Gabelas. La relación entre estudiantes es un aprendizaje de pares con el acompañamiento del coordinador y tutor-profesor. Se evidencia así una línea horizontal de trabajo donde la reciprocidad es sustancial, realizando "énfasis en el proceso" (Kaplún, 1998). Los estudiantes que conforman la redacción, editor y jefe de sección, más el profesor que los coordina y el público intercambian los roles de emisor y receptor, en una dinámica de producción interactiva, que ejemplifica la naturaleza educomunicativa de la plataforma.

"Entremedios" significa conexión con todas y cada una de las secciones (EntrePlanos, EntreVips, EntreLíneas, EstreSpots, EntreOndas, EntreEventos y EntreLinks), secciones que contienen la diversidad de los medios que conforman el periodismo. Una red de secciones con diferentes temáticas pero conectadas en sus dinámicas de trabajo y en la retroalimentación de sus contenidos. Por consiguiente, el valor psicosocial y el valor sináptico que definen el Factor Relacional, formalizan el desarrollo de esta plataforma.

El respeto que todo buen periodista debe tener hacia su público será en este curso el leit motiv de la plataforma, con el eslogan que marcó el inicio del 20202021: "Los periodistas del futuro hacen el periodismo de ahora". Los estudiantes tienen la oportunidad de ejercer como buenos profesionales porque sus contenidos no están dirigidos a sus compañeros y compañeras, tampoco a otros estudiantes ni a otras universidades, sino a la sociedad, que merece y necesita la mejor información y la mejor opinión. Por este motivo, priorizamos en este curso la calidad del producto. 
Solo se publicarán las piezas que cumplan con rigor los indicadores de calidad, revisados por el jefe de sección, el editor y supervisados por el profesor coordinador de sección, según las directrices que marca el libro de estilo de este medio de comunicación digital.

\section{RESULTADOS}

Con objeto de analizar los motivos del cambio de orientación y fortalecimiento de la plataforma Entremedios para convertirse en un medio atractivo para la formación de los estudiantes de periodismo, a través de la práctica periodística de calidad con fines de transferencia de conocimiento, aplicamos las técnicas del grupo de discusión a egresados y las entrevistas en profundidad a profesorado responsable. A partir de los resultados, se trazó el cambio que hemos comentado en el curso académico 2020-2021.

\section{Análisis del grupo de discusión}

Tras llevar a cabo el grupo de discusión entre los diferentes participantes, en un tono cercano y relajado, acerca de la plataforma del Grado en Periodismo, la idea principal y unánime que tienen todos los egresados que participaron en el grupo de discusión es que el proyecto necesita redefinirse.

En la página web se especifica que su objetivo principal es el de "visibilizar las prácticas que han realizado las alumnas y alumnos durante su proceso de aprendizaje." Sin embargo, los antiguos alumnos, tras navegar por las diferentes secciones y contenidos disponibles, opinan que, si bien sí que aparecen prácticas de diferentes asignaturas, lo que predomina en "Entremedios" son noticias cortas sobre acontecimientos relacionados con el Grado en Periodismo, ya sea la publicación del libro de algunos profesores o premios ganados por algunos de los alumnos. Por este motivo, los egresados consideran que la plataforma está a medio camino entre repositorio de prácticas y medio de información y comunicación y, de esta manera, la plataforma no funciona del todo ni resulta excesivamente atractiva. En su opinión, "Entremedios" y todos sus integrantes deberían centrarse en publicar únicamente aquellos trabajos académicos que se consideren adecuados y que puedan suponer un plus para el futuro periodista, es decir, la que fuera la idea original para la web periodismo.unizar.es.

La opinión general de los participantes en el grupo de discusión es que, como medio de comunicación no va a resultar competitivo frente a otros medios, ya que se encuentra enormemente ligado a la universidad. Consideran que casi todos los temas versan sobre aspectos institucionales, lo que limita excesivamente el público objetivo.

Por otra parte, para funcionar como medio, la plataforma necesitaría de una periodicidad de publicación y una actualización constante que no posee ${ }^{2}$. Sin 
embargo, los antiguos alumnos, tras debatir los diferentes usos que se le podía dar a una web de estas características, consideran su creación como repositorio muy positiva, ya que funciona como escaparate y presentación de las habilidades de los futuros profesionales de la comunicación. El tener un lugar al que poder acceder para presentar diferentes trabajos propios y proyectarlos hacia el exterior genera un valor añadido a los currículums de los estudiantes. También es muy valorable como lugar al que pueda acceder el futuro alumnado de Periodismo para conocer más de cerca algunas de las tareas que ellos mismos realizarán a posteriori. No obstante, consideran que para que esta plataforma genere ese valor añadido debe dársele una difusión adecuada y hasta el momento, en su opinión, no se ha conseguido. Casi todos los participantes del grupo de discusión salvo cuatro, desconocían hasta el momento de llevar a cabo la práctica la existencia de "Entremedios" y lo achacan a una escasa publicidad de la plataforma.

Algunas de sus ideas para mejorar la visibilidad del medio fueron aceptadas y consideradas bastante interesantes por todos los debatientes. Más allá de nutrir constantemente las redes sociales, sobre todo dando una gran importancia a una cuenta de Instagram o Flickr donde publicar fotoperiodismo, los egresados opinan que la realización de un evento llamativo, para que medios locales publiquen una pequeña nota en sus propias páginas web y redes sociales sobre esta nueva plataforma y la gente escuchase el nombre de "Entremedios", podría suponer un aumento muy grande de visitantes para la web. Otro detalle que consideran importante es la necesidad de un canal propio en Youtube. Se puede comprobar que aquellas entradas que cuentan con vídeos o informaciones audiovisuales están subidas a la plataforma de streaming desde la cuenta de la Facultad de Filosofía y Letras, por lo que sus vídeos e informaciones se mezclan en Youtube con otros de muy distinta índole.

Mediante pequeñas acciones como estas, "Entremedios" podría incrementar su presencia en la red y generar más visitas. Una ventaja enorme con la que parte la plataforma es su posicionamiento en Google. Se ha buscado a través de una sesión de incógnito el nombre de la web y aparecía en primer lugar en las búsquedas del servidor y pocas veces ocurre algo así, especialmente teniendo en cuenta que la web no cuenta con dominio propio, por lo que, los egresados califican de inmejorable este aspecto de "Entremedios".

Otra de las opiniones generalizadas surgida en el grupo de discusión es que, estéticamente, la página web resulta atractiva y profesional, lo que puede aumentar el tiempo de permanencia en la plataforma de los usuarios. No obstante, los participantes consideran que los nombres elegidos para las secciones no son acertados y provocan confusión en aquellas personas que visitan la página. A su modo de ver, una persona no busca contenidos según su formato de creación -ya sea vídeo, audio o prensa- sino por temáticas -televisión, deporte, política...-. Es más sencillo encontrar temas de interés de esta manera y relegar el formato a meras etiquetas o hashtags. Si bien jugar con el nombre de la plataforma en las nomenclaturas de las diferentes secciones es ingenioso, no resulta efectivo a la hora de estructurar las informaciones. 
Además, otro aspecto mejorable que destacan los participantes es que algunas de estas categorías se encuentran vacías, sin ningún tipo de publicación en ellas, lo que, para ellos, puede generar una opinión desfavorable en el usuario. Opinan que esto podría mejorarse con una mayor implicación del alumnado. Creen que podría llegar a ser positivo, tanto para la plataforma como para los estudiantes, ligar este proyecto con algunas asignaturas de la carrera, como "Proyecto de comunicación digital" (asignatura obligatoria de $4^{\circ}$ curso del Grado en Periodismo, 9 Cr. ECTS). Así, todo el alumnado, curso tras curso, participaría en el desarrollo de "Entremedios" y a su vez, de este modo, podría nutrirse de contenidos más asiduamente. Otra posibilidad sería que un número determinado de publicaciones supusieran la convalidación de la asignatura optativa "Prácticum" (6 Cr. ECTS), como ocurre con la participación en la radio de la Universidad (Radio Unizar). De hecho, muchos de los participantes comparan ambos medios y los encuentran muy similares, sobre todo con la radio de los inicios, la cual se centraba estrictamente en temas concernientes a la universidad y, tras abrir sus miras y ampliar temáticas, además de aumentar su presencia en redes sociales, se generó un aumento de oyentes considerable.

A su vez consideran que el hecho de abandonar las noticias cortas de actualidad inmediata en el tiempo que quedan desfasadas con rapidez, y ahondar en temas de interés propio del estudiantado, con géneros descriptivos y más atemporales pero que siempre cuenten con un gancho de interés, también podría mejorar el estímulo de los estudiantes para publicar en "Entremedios". Sobre todo, los egresados destacan que las generaciones más jóvenes -alumnos de primero y segundo curso, principalmente- deberían contar con más participación dentro del medio ya que son ellos los que "se encuentran con más fuerza y motivación por la profesión y la carrera”.

En resumen, los participantes valoran como positiva la creación de este medio siempre y cuando se realicen las mejoras que, objetivamente, ellos consideran oportunas para que así destaque como plataforma universitaria dentro de las posibles competidoras que puedan existir en otros campus universitarios y siga manteniendo un alto reconocimiento a nivel nacional.

\section{Análisis de las entrevistas en profundidad}

La idea central obtenida como resultado de las entrevistas es que "Entremedios" supone el salto de TRICLab, el laboratorio de ideas fundado por el grupo de investigación GICID y en el que participan investigadores y académicos nacionales e internacionales bajo el nombre de "TRIClabers", a un medio real, una plataforma de comunicación en la que esta idea, la de comunicar, es la central.

Los entrevistados destacan que la plataforma cuenta con un elemento diferenciador respecto al resto de medios de comunicación al uso: la defensa de que las TIC, lejos de separar al ser humano, pueden permitir que este se relacione de una 
forma más eficiente y rápida si se usan con conocimiento. De ahí la "R" del factor "Relacional" añadida a las Tecnologías de Información y Comunicación.

Su carácter innovador y transmedia lleva a "Entremedios" a buscar un público objetivo muy definido, segmentado por intereses y comportamientos. Es decir: gente de distintos ámbitos profesionales que pueda estar interesada en la metodología de trabajo TRIC y en temas de interés general; pero que también esté dispuesta a tener un papel activo en la plataforma, que servirá de punto de reunión para conversar sobre distintos temas. Esto último requerirá de un mayor desarrollo en el futuro inmediato.

Con la vista puesta en ese futuro cercano, ambos entrevistados destacan que se tendrá en cuenta tanto la integración de distintas herramientas multimedia que permitan presentar cada noticia utilizando el recurso más apropiado, como la necesaria reciprocidad entre emisor y receptor, del que se esperará su involucración para enriquecer el propio contenido de las noticias. De ahí que "Entremedios" no busque un hueco en el mercado mediático tradicional y se defina como un medio especializado: la plataforma del Grado en Periodismo de la Universidad de Zaragoza.

Por otro lado, debido a su espíritu innovador, "Entremedios" sigue una filosofía de trabajo diferenciadora, centrada en dos ámbitos, a juicio de los entrevistados: 1. Reducir, e incluso eliminar, ese miedo a las TIC, enseñando que estas, bien utilizadas, pueden permitir al ser humano informarse y comunicarse de forma más rápida y eficiente; y 2. En su propósito de proporcionar una información de calidad, desde la plataforma se tiene un carácter examinador y crítico con todo lo que se publica. En etapas subsiguientes, otro elemento diferenciador será la capacidad de creación y actualización cada vez más inmediata, objetivo para el que se hará uso de distintas herramientas multimedia y se tratará de enfocar las noticias empleando aquellos recursos que mejor se adapten a su contenido.

En definitiva, se trata de un proyecto ambicioso que cuenta con varios elementos clave para aunar aprendizaje multimedia y transferencia de conocimiento, debido a que:

1. Su enfoque es distinto en todos los sentidos al de los medios de comunicación tradicionales.

2. Su filosofía de trabajo es novedosa y podría llegar a suponer un cambio en la forma de comunicarnos en la era digital pero sin renunciar al factor humano y relacional.

3. La audiencia a la que estará dirigido es muy específica.

4. Los temas que se abordarán no serán los mismos que se tratan en los medios generalistas, y estarán abiertos al debate e interacción; una forma de asegurar el enriquecimiento y la puesta al día constante de la plataforma.

5. La herramienta es un ejercicio proactivo de estudiantado y profesorado que propicia la difusión de las actividades prácticas realizadas por el alumnado, potenciando así su proyección pública como futuros periodistas. 


\section{DISCUSIÓN Y CONCLUSIONES}

El aprendizaje multimedia constituye una notable oportunidad para los procesos formativos de diversa índole. Las Tecnologías de la Relación, la Información y la Comunicación (TRIC) están generando un profundo impacto en la forma en que nos relacionamos, aprendemos y accedemos a la información, afectando así a todas las esferas de la vida. El estudiantado que forma parte de las nuevas generaciones se encuentra inmerso en un mundo plenamente interconectado y posee diferentes maneras de relacionarse y también de aprender. El creciente uso de las tecnologías digitales para la enseñanza en la educación superior pretende ser una respuesta a esa necesaria adaptación del proceso educativo a los nuevos discentes y a sus nuevos escenarios, aunque en muchas ocasiones se limita a un recurso de apoyo para un proceso formativo que sigue anclado en los mismos parámetros.

Como plantea Silva (2017), los entornos virtuales de aprendizaje requieren el uso de metodologías centradas en el alumno y la incorporación de las TIC para compartir y distribuir conocimiento en red. En el contexto universitario, debe ir más allá del simple traspaso del modelo formativo convencional al espacio virtual.

Convenimos con Bidarra y Rusman (2017) que el aprendizaje multimedia, mediante la creación de historias digitales mejora las habilidades de manejo de una variedad de herramientas multiformato, ya que permiten operar con texto, imágenes fijas, audio, vídeo y publicación en la web. De este modo, se optimiza el proceso de aprendizaje, dotando al estudiantado de un rol de productor de contenidos, lo que les empodera e implica de manera real. Asimismo, como aborda Ossorio-Vega (2014), se refuerza su capacidad crítica y su autosuficiencia, al permitirles seleccionar el camino a seguir.

Los espacios virtuales de aprendizaje precisan de nuevos roles y perfiles docentes. La tutorización y la dinamización se convierten en claves para el adecuado seguimiento del proceso de aprendizaje, como también lo son el diseño de contenidos didácticos adaptados y la creación de dinámicas pedagógicas de carácter virtual. En este sentido, la adquisición de competencias mediáticas digitales es una necesidad cada vez más irrenunciable para el profesorado, pues son conocimientos que es necesario transmitir al alumnado. Un alumnado, a su vez, cada vez más empoderado, que es protagonista y creador de su propio proceso de aprendizaje y que es capaz de crear contenidos que, como ocurre en la plataforma "Entremedios", sirven y retroalimentan también el proceso de aprendizaje de otros.

"Entremedios" se define como un medio especializado de publicación de las mejores prácticas del estudiantado del Grado en Periodismo de la Universidad de Zaragoza, se apuesta más por la integración de contenidos con una exigencia de calidad, revisados por profesionales y académicos.

Los egresados que participaron en la plataforma en sus primeros años de puesta en marcha califican la experiencia de positiva, pero mejorable. Exponen una serie de aspectos mejorables que son considerados por los responsables y encaminan 
un conjunto de reformas, ya impulsadas en su mayoría desde la coordinación. Este feedback ha resultado fundamental para la optimización de la plataforma.

Si bien la puesta en marcha de Entremedios adoptó una fórmula excesivamente académica, en el último curso, atendiendo a los aspectos mejorables planteados por los egresados, se han ido llevando a cabo con objeto de profesionalizarla en pro de la calidad del medio, en cuanto a periodicidad, actualización constante, número de entradas, géneros más atemporales, piezas más elaboradas y calidad de las mismas.

Además, en el último curso, también han comenzado a participar alumnos y alumnas de primer curso, para dar respuesta a la propuesta de los egresados y comenzar desde el inicio de carrera la posibilidad de publicar en el medio.

El carácter relacional de "Entremedios", con la aplicación de la metodología de trabajo TRIC, posibilita que los usuarios tengan un rol activo mayor y puedan conversar sobre los distintos temas que se publican. Los responsables plantean un mayor desarrollo futuro de estas interacciones.

El Factor Relacional ha cristalizado en este proyecto ya consolidado. Su dinámica de trabajo en la que cada sección reproduce lo que es la redacción de un medio, capacita a los estudiantes en competencias profesionales que reclama el mercado de la comunicación. La estructura relacional que garantiza "Entremedios" permite la fluidez de los contenidos, la permeabilidad de los formatos, la hibridación de los lenguajes. Los fundamentos humanísticos y tecnológicos ya indicados, que avalan el Factor Relacional, dotan esta plataforma multimedia de los estudiantes y con los estudiantes, de una ventana abierta al público y sus audiencias.

El hecho de que la plataforma cuente con un excelente posicionamiento en Google, puesto que aparece en primer lugar en las búsquedas del servidor, supone un aliciente para el estudiantado, si bien se plantean otras formas de difusión complementaria, por ejemplo, a través de un canal de Youtube de los vídeos realizados.

El carácter de repositorio de contenidos que funciona como escaparate de las mejores actividades del alumnado sirve para proyectar las competencias de los futuros profesionales de la comunicación hacia el exterior, lo que supone un valor añadido para los currículums de los estudiantes y un modelo de transferencia de conocimiento hacia la sociedad.

Este estudio puede servir de referencia para futuras investigaciones relacionadas con el aprendizaje multimedia a través de plataformas digitales, dada la aplicación del componente práctico que ese tipo de entornos de aprendizaje universitario tienen, a lo que se añade la dimensión relacional, más allá del uso de la tecnología y de la producción de contenidos. Como futura línea de investigación, se plantea analizar las formas de difusión complementaria, a través de redes sociales, que sirven para ampliar la divulgación de la información contenida en el medio digital $\mathrm{y}$ dar a conocer en mayor medida la labor profesional que ejercen los estudiantes. 


\section{NOTAS}

1. I Simposio "La brecha digital entre generaciones en la escuela y el hogar" https://www.url.edu/es/sala-de-prensa/agenda/ferias-congresos-yexposiciones/2012/i-simposio-del-grupo-de-investigacion-conincom-austica-sobrela-brecha-digital-entre-generaciones-en-la-escuela-y-el-hogar-en-la-fcc-blanquerna

2. Tras la realización del grupo de discusión, la plataforma ha experimentado una reorganización que ha aumentado la sistematicidad de las publicaciones, así como su periodicidad (una al día, repartidas entre las distintas secciones), y la homogeneización estética y formal a través de la promoción del libro de estilo de la web.

\section{REFERENCIAS}

Álvarez, C., y San Fabián, J. L. (2012). La elección del estudio de caso en investigación educativa. Gazeta de Antropología, 28(1). https://doi. org/10.30827/Digibug.20644

Angulo, J. F., y Vázquez, R. (2003). Introducción a los estudios de casos. Aljibe.

Bidarra, J., y Rusman, E. (2017). Towards a pedagogical model for science education: bridging educational contexts through a blended learning approach. Open Learning: The Journal of Open Distance and e-Learning, 32(1), 6-20. https://doi. org/10.1080/02680513.2016.1265442

Bullen, M., y Morgan, T. (2015). Digital Learners in Higher Education: Implications for Teaching, Learning \& Technology. En M. Gisbert y M. Bullen (Eds.), Teaching and Learning in Digital Worlds: Strategies and Issues in Higher Education (pp. 11-19). Mercè Gisbert y Mark Bullen.

Castells, M. (2006). La Sociedad Red. Una visión global. Alianza Editorial.

Cebreiro López, B., y Fernández Morante, M. C. (2004). Estudio de casos. En F. Salvador Mata, J. L. Rodríguez Diéguez y A. Bolívar Botía. Diccionario enciclopédico de didáctica. Aljibe.

Cerdeño-Romero, E. (2019). Entornos virtuales de aprendizaje y su rol innovador en el proceso de enseñanza. ReHuSo: Revista de Ciencias Humanísticas y Sociales, 4(1), 119-127. https://doi. org/10.33936/rehuso.v4i2.1845

Coller, X. (2005). Estudio de casos. Centro de Investigaciones Sociológicas.

Dziuban, Ch., Grahan, Ch. R., Moskal, P., Norberg, A., y Sicilia, N. (2018). Blended learning: the new normal and emerging technologies. International Journal of Educational Technology in Higher Education, 15(3), 1-16. https://doi. org/10.1186/s41239-017-0087-5

Gabelas-Barroso, J. A, Marta-Lazo, C., y Hergueta Covacho, E. (2013). El Factor R-elacional como epicentro delas prácticas culturales digitales. En D. Aranda, J. Sánchez Navarro (Eds). Educación, Medios y Cultura de la participación (pp. 351-374). Editorial UOC.

Gabelas-Barroso, J. A., y Marta-Lazo, C. (2017). Habitaciones de Cristal. Blog Educar en Comunicación. http:// educarencomunicacion.com/

Gillespie, T. (2010). The politics of plataforms. New Media Society. https:// doi.org/10.1177/1461444809342738

Gisbert, M., y Johnson, L. (2015). Educación y tecnología: nuevos escenarios de aprendizaje desde una visión transformadora. Universities and 
Knowledge Society Journal, 12(2), 1-14. https://doi.org/10.7238/rusc.v12i2.2570

Hurlbut, A. R. (2018) Online vs. traditional learning in teacher education: a comparison of student progress. American Journal of Distance Education, 32(4), 248-266. https://doi.org/10.1080/ 08923647.2018.1509265

Jenkins, H. (2007). Convergence Culture; Where Old a New Media Collide. UOC.

Kaplún, M. (1998). Una pedagogía de la comunicación. Ediciones De la Torre.

Marta-Lazo, C., y Gabelas, J. (2016). Comunicación Digital. Un modelo basado en el Factor R-elacional. UOC.

Marta-Lazo, C., Hergueta-Covacho, E., y Gabelas-Barroso, J. A. (2016). Applying Inter-methodological Concepts for Enhancing Media Literacy Competences. Journal of Universal Computer Science, 22(1), 37-54. https://bit.ly/3dkJ94L

Mayer, R. E. (2018). Thirty years of research on online learning. Applied Cognitive Psychology, 33(2), 152-159. https://doi. org/10.1002/acp.3482

Murray, D. (2020). La masa enfurecida. Península.

Nennig, H. T., Idárraga, K. L., Salzer, L. D., Bleske-Rechek, A., y Theisen, R. M. (2020). Comparison of student attitudes and performance in an online and a face-to-face inorganic chemistry course. Chemistry Education Research and Practice. https://doi.org/10.1039/ C9RPo0112C

Ossorio-Vega, M. A. (2014). Application of Transmedia Storytelling in Spanish Universities:Collaborative Learning, Multi platform and Multi format. Internacional Technology, Science and Society Review, 3(2), 25-38. https://doi. org/10.37467/gka-revtechno.v3.1186

Saavedra-Bautista, C., Cuervo-Gómez, W. O., y Mejía-Ortega, I. D. (2017). Producción de contenidos transmedia, una estrategia innovadora. Revista Científica, 28(1), 6-16. $\quad$ https://doi.org/10.14483/ udistrital.jour.RC.2017.28.a1

Salinas, J. (2004). Innovación docente y uso de las TIC en la enseñanza Universitaria. Universidad y Sociedad del Conocimiento, 1(1). https://doi. org/10.7238/rusc.v1i1.228

Silva, J. (2017). Un modelo pedagógico virtual centrado en las E-actividades. Revista de Educación a Distancia, 17(53), 1-20. https://doi.org/10.6018/red/53/10

Van Dijk, J. (2016). La cultura de la conectividad. Una historia de las redes sociales. Siglo Veintiuno Editores.

\section{PERFIL ACADÉMICO Y PROFESIONAL DE LOS AUTORES}

Carmen Marta-Lazo. Catedrática de Periodismo de la Universidad de Zaragoza. Investigadora Principal del Grupo de Investigación en Comunicación e Información Digital (GICID). Directora de Radio.Unizar y de la plataforma digital Entremedios. Presidenta de Investigación de la Asociación de Radios Universitarias de España (ARU). Co-editora de Revista Mediterránea de Comunicación. Ha escrito más de doscientas publicaciones, entre libros, capítulos y artículos relacionados con sus líneas de investigación: educación mediática, competencias digitales y usos e interacciones en redes sociales. En Google Scholar cuenta con un índice H de 25: https://scholar.google.es/citations?user=vxisuHoAAAAJ\&hl=es. Y en ORCID con 207 publicaciones: https://orcid.org/0000-0002-0004-1094. En ResearchGate con 889 citaciones: https://www.researchgate.net/profile/Carmen-Lazo-2. Y en Dialnet 
es la autora mujer más citada en el área de Periodismo: https://dialnet.unirioja.es/ servlet/autor?codigo $=2308979$. https://orcid.org/0000-0002-0004-1094 E-mail: cmarta@unizar.es

José Antonio Gabelas-Barroso. Profesor Titular de la Unidad Predepartamental de Periodismo y Comunicación Audiovisual y Publicidad de la Universidad de Zaragoza. Doctor en Ciencias de la Información. Licenciado en Historia del Arte. Diplomado en Cinematografía. Diplomado en Profesorado de Educación General Básica. Director de la colección "Comunicación y Medios" 19982008 en Prensa Diaria Aragonesa. Miembro fundador del grupo GICID (Grupo Investigación en Comunicación e Información Digital), Universidad de Zaragoza. Director de la plataforma multimedia "Entremedios" del Grado de Periodismo de la Universidad de Zaragoza. Miembro del Consejo de Redacción del medio de opinión Disidentia.com. @jgabelas. https://orcid.org/0000-0002-5942-6140

E-mail: jgabelas@unizar.es

Antonia Nogales-Bocio. Profesora Contratada Doctora de la Unidad Predepartamental de Periodismo y Comunicación Audiovisual y Publicidad de la Universidad de Zaragoza. Doctora y Licenciada en Periodismo por la Universidad de Sevilla. Máster en Filosofía y Cultura Moderna por la Universidad de Sevilla. Presidenta del Laboratorio de Estudios en Comunicación: https://ladecom. es. Miembro de los grupos de investigación GICID (Grupo Investigación en Comunicación e Información Digital) de la Universidad de Zaragoza, GREHCCO (Grupo Investigación en Estructura, Historia y Contenidos de la Comunicación) en la Universidad de Sevilla, y SMEMIU, adscrito a la UNED. https://orcid.org/oooo0003-0050-6979

E-mail: anogales@unizar.es

Miguel Ezequiel Badillo-Mendoza. Doctor en Comunicación y educación en entornos digitales, docente asistente de la Universidad Nacional Abierta y a Distancia, UNAD, Colombia. Fue Secretario Académico de la Asociación Colombiana de Investigadores, ACICOM, (2016-2020) co-director del grupo de investigación FISURA, investigador del GICID Universidad de Zaragoza, e Investigador Junior en Colciencias. Fue Líder Nacional de la Cadena de Formación en Comunicación Social de la UNAD (2014-2019), coordinador de la Cátedra de Comunicación UNAD (2014, 2016, 2017 y 2018) e integrante de la comisión nacional intersectorial de aseguramiento de la calidad se la educación superior -CONACES-. https://orcid. org/0000-0003-2247-3864

E-mail: miguel.badillo@unad.edu.co 
Fecha de recepción del artículo: 15/05/2021

Fecha de aceptación del artículo: 23/08/2021

Fecha de aprobación para maquetación: 06/09/2021 